\title{
Effect Of Surgical Safety Checklists On Gastric Cancer Outcomes: A Single-Center Retrospective Study
}

\section{Deliang Yu \\ Qingchuan Zhao}

Department of Digestive Surgery, Xijing Hospital of Digestive Diseases, Air Force Military Medical University, Xi'an, Shaanxi 710032, People's Republic of China
Correspondence: Qingchuan Zhao Department of Digestive Surgery, Xijing Hospital of Digestive Diseases, Air Force Military Medical University, 127 West Changle Road, Xi'an, Shaanxi 7I0032,

People's Republic of China

Tel +86-2915339297569

Email zhaoqcxjh@I63.com
This article was published in the following Dove Press journal: Cancer Management and Research

\begin{abstract}
Aims: Surgery is the primary treatment option for patients with gastric cancer, however the rate of postoperative complications are still high. The implementation of surgical safety checklists (SSCs) has been shown to reduce morbidity and mortality. This study aimed to evaluate the effect of SSCs on the clinical outcomes of gastric cancer.

Methods: A total of 881 gastric cancer patients who underwent D2 gastrectomy from May 2009 to April 2011 in a large teaching hospital in China were included in this retrospective study. Patients were matched and divided into the control group (SSC nonimplementation) and intervention group (SSC implementation). The outcomes including intraoperative condition, postoperative complications, and prognosis were then compared between the groups. Results: The control group comprised 414 patients (47.0\%), and the intervention group included 467 patients $(53.0 \%)$. Patients in the intervention group had a significantly shorter length of postoperative stay $(\mathrm{P}<0.001)$. Operation time, blood loss, blood transfusion, and hospital charges were comparable between the two groups (all P >0.05). SSC was not associated with postoperative complications (all $\mathrm{P}>0.05$ ). Overall survival was also comparable between patients in the two groups $(\mathrm{P}>0.05)$.

Conclusion: The implementation of an SSC was associated with a decreased length of postoperative stay in gastric cancer patients following D2 gastrectomy but did not significantly affect the other outcomes.
\end{abstract}

Keywords: gastric cancer, surgical safety checklist, prognosis, medical quality

\section{Introduction}

Gastric cancer is the fifth most frequently diagnosed cancer and the third leading cause of cancer-related death around the globe. ${ }^{1}$ China currently has the highest number of newly diagnosed gastric adenocarcinoma patients in the world. ${ }^{2}$ Surgery is the primary treatment option for patients with gastric cancer and more than 100,000 gastric cancer surgeries are performed each year in China alone. ${ }^{3}$ The rate of postoperative complications for gastric cancer surgeries has been estimated at $15.2 \%{ }^{4}$ Therefore, postsurgical care and attendant complications represent a substantial burden that is deserving of attention from the public health community.

Hospitals around the world use surgical safety checklists (SSCs) for various operations. The implementation of a 19-item SSC designed by the World Health Organization (WHO) has been shown to reduce the numbers of deaths and complications. ${ }^{5}$ Research on the value of SSCs has gradually been increasing. However, there is debate about their role in reducing postoperative complication rates, with some studies reporting that SSCs 
have no effect on postoperative complication rates, ${ }^{6,7}$ whereas others report an increase on postoperative complication rates. ${ }^{8,9}$ The associations between SSC and the clinical outcomes of several specific surgeries have been investigated, including podiatric surgery ${ }^{10}$ and neurosurgery. ${ }^{11}$ However, the association between SSCs and gastric cancer surgeries has not yet been reported. Thus, the present study aimed to evaluate the effect of SSCs on the clinical outcomes of gastric cancer.

\section{Materials And Methods}

This retrospective observational study was performed in a large teaching hospital in China. All patients provided written informed consent for review of their medical records, in accordance with the Declaration of Helsinki. The Ethics Committee of Xijing Hospital approved all protocols.

A consecutive series of gastric cancer patients undergoing D2 gastrectomy were screened from May 2009 to April 2011; the exclusion criteria included any preoperative neoadjuvant therapy, treatment with other viscera resection, distant metastasis, and confirmation of nonadenocarcinoma after the operation. Figure 1 shows the flow chart of patient enrolment. The surgeries included total, proximal, and distal D2 gastrectomy. Surgical procedures and postoperative chemotherapy were performed according to the recommendations of the Japanese Gastric Cancer Treatment Guidelines ${ }^{12}$ and the NCCN guidelines for gastric cancer.

An SSC was implemented at the hospital starting from May 1, 2010. Patients were divided into two groups: a control

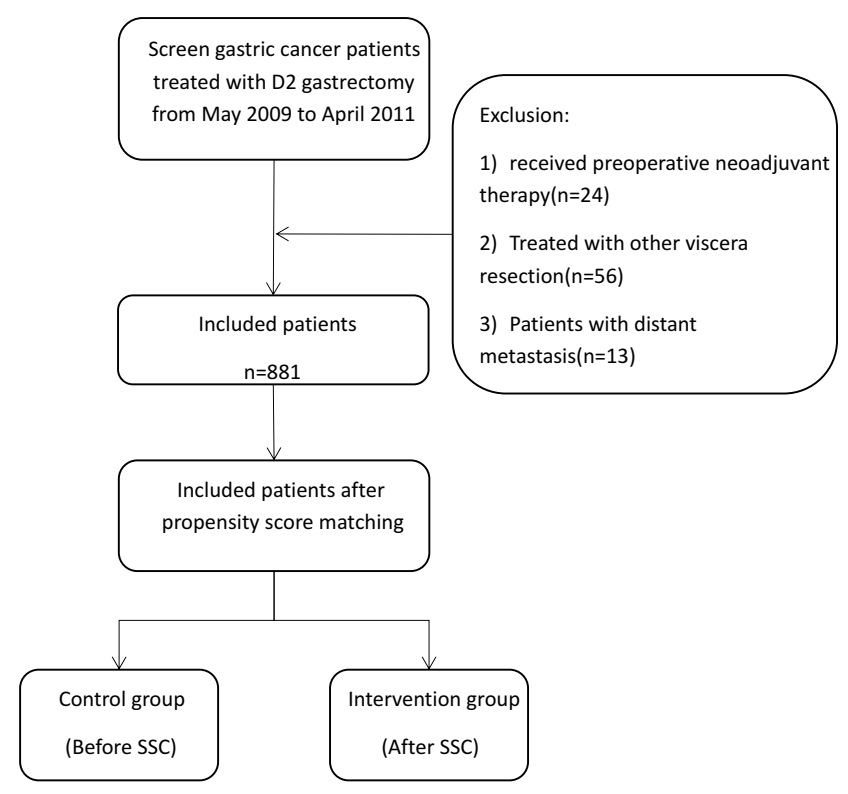

Figure I Study flow chart. group that underwent surgery before SSC implementation and an intervention group that underwent surgery after SSC implementation.

All patient data were anonymized for this study. Clinicopathological data including gender, age, body mass index (BMI), smoking, comorbidities (including hypertension, coronary heart disease, and diabetes mellitus), ASA classification, operative approach, type of gastrectomy, tumor location, tumor size, differentiation grade, tumor depth, lymph node metastasis (LNM), and tumor stage were collected. Surgery-related data including blood loss, operation time, blood transfusion, length of postoperative stay, and hospital charges were recorded. Postoperative complications within 30 days, including pneumonia, ileus, anastomosis leakage, abdominal bleeding, postoperative abdominal infection, wound complication (e.g., wound disruption and wound infection), unplanned reoperation, and mortality were recorded via telephone calls and outpatient follow-up. Patients were followed up every 3 months until May 2016.

To reduce bias, propensity score matching (PSM) was performed using the following parameters: gender, age, BMI, smoking, comorbidities, ASA classification, operative approach, type of gastrectomy, tumor location, tumor size, differentiation grade, tumor depth, LNM, and tumor stage. We estimated the PSM models using one-to-one nearest-neighbour matching techniques without replacement and with a 0.01 caliper level. Each participant with SSC implementation was matched to another participant without SSC implementation in this cohort and the propensity scores for the two could be no farther than 0.01 apart. If the matching procedure was successful, there would be no differences on the covariates between the two groups (achieving covariate balance).

After matching procedure, we assessed whether there was an association between surgery-related parameters, postoperative complication and SSC implementation, and we also did the univariate analysis of risk factors for postoperative complications of gastric cancer. In the last step of the analysis, we assessed overall survival of gastric cancer patients stratified by SSC before and after matching procedure.

Data were processed using SPSS 22.0 for Windows (SPSS Inc., Chicago, IL, USA), the statistical software packages R (http://www.R-project.org, The R Foundation) and EmpowerStats (http://www.empowerstats.com, X\&Y Solutions, Inc., Boston,MA). Continuous variables were expressed as median $\pm \mathrm{SD}$ and analyzed using nonparametric 
tests. Age, BMI, and tumor size were translated into categorical data. Categorical data were cross-tabulated, and differences in proportions were tested using chi-squared (X2) or Fisher's exact tests. Significant prognostic risk factors were assessed by univariate analysis, and overall survival was analyzed by the Kaplan-Meier method. A P value was considered statistically significant at or below the $5 \%$ level.

\section{Results}

A total of 1046 gastric cancer patients were screened. Among these patients, 165 patients were excluded, including 24 patients who received preoperative neoadjuvant therapy, 56 patients treated with other viscera resection, 13 patients with distant metastasis, and 72 patients confirmed as nonadenocarcinoma after operation. Therefore, a total of 881 patients served as the study population.

The study population included 694 male patients $(78.8 \%)$ and 187 female patients $(21.2 \%)$, with 414 patients $(47.0 \%)$ in the control group and 467 patients $(53.0 \%)$ in the intervention group. The median age was $57.2 \pm 11.5 y e a r s$ old. The median follow-up durations of the control and intervention groups were $41.9(0-85)$ and $34.8(0-85)$ months, respectively. There were no differences with regard to gender, age, BMI, smoking, comorbidities, tumor location, or tumor size in the two groups (all $\mathrm{P}>0.05$ ). There were significant differences in ASA classification, operative approach, type of gastrectomy, differentiation grade, tumor depth, LNM, and tumor stage (all $\mathrm{P}<0.05$ ). Thus, propensity score matching was used to match patients in the two groups to reduce bias. After matching, there were 325 patients each in the two groups. After matching, the clinicopathological features were comparable among the two groups after matching (all $\mathrm{P}>0.05$ ). Table 1 shows the associations between clinicopathological characteristics and SSC implementation or nonimplementation before and after matching.

Table 2 shows the association between SSC implementation or nonimplementation and surgery-related parameters. The results showed that patients in the intervention group were associated with decreased length of postoperative stay $(9.64 \pm 2.67$ vs $11.02 \pm 3.30, \mathrm{P}<0.001)$. Operation time, blood loss, blood transfusion, and hospital charges were comparable between the two groups (all $\mathrm{P}>0.05$ ).

Table 3 shows the association between SSC implementation or nonimplementation and postoperative complications. The results showed that SSC implementation or nonimplementation was not associated with complications (all P > 0.05).

The risk factors for postoperative complications of gastric cancer were analyzed using univariate analysis
(Table 4). Hypertension, LNM, and tumor stage were significantly associated with postoperative complications of gastric cancer.

Figure 2A shows the overall survival of gastric cancer patients stratified by SSC before propensity score matching. The overall survival was comparable between patients in two groups $(\mathrm{P}=0.239)$. After matching (Figure $2 \mathrm{~B})$, the overall survival was still comparable between the two groups $(\mathrm{P}=0.453)$.

\section{Discussion}

Surgical complications represent a significant cause of morbidity and mortality, with the rate of major complications after inpatient surgery estimated at $3-17 \%$ in industrialized countries. ${ }^{13,14}$ SSCs may prevent errors, deaths, and complications during surgery or perioperatively. A variety of interventions have shown promise for improving patient safety and shortening the length of hospital stay. However, opposition still exists. The present study was the first study to investigate the effect of SSC on the clinical outcomes of gastric cancer after radical gastrectomy. We found that SSC implementation was associated with a decreased length of postoperative stay but had no effect on operation time, blood loss, blood transfusion, hospital charges, or postoperative complications and had no relation to the overall survival of gastric cancer patients.

Differences did exist before and after SSC implementation in ASA classification, operative approach, type of gastrectomy, differentiation grade, tumor depth, LNM, and tumor stage. However, given that it is only a safeguard for surgical safety, SSC has no logical relationship with these indexes. Nevertheless, to reduce bias, propensity score matching was performed in this study.

There are several previous reports on the associations between SSC and intraoperative condition. Papaconstantinou et $\mathrm{al}^{15}$ reported that the implementation of a SSC did not negatively affect the operation time. Lacassie et $\mathrm{al}^{16}$ reported that the median length of stay for adult surgery patients decreased after the implementation of the WHO SSC. Similar to the present study, they also found no association between SSC and blood loss, transfusion, or hospital charges. To date, there is no evidence that SSC can help reduce hospital charges.

Previous studies have shown that SSCs can reduce postoperative complications. However, previous works did not assess the postoperative complications of gastric cancer. In the current study, we did not find any evidence that $\mathrm{SSC}$ decreased the rate of postoperative complications 
Table I Correlations Between Clinicopathological Characteristics And SSC Implementation Or Nonimplementation Before And After Matching

\begin{tabular}{|c|c|c|c|c|c|c|c|}
\hline & & \multicolumn{3}{|c|}{ Before Matching $(n=88 I)$} & \multicolumn{3}{|c|}{ After Matching $(n=650)$} \\
\hline & & $\begin{array}{l}\text { Control } \\
\text { Group } \\
(n=414)\end{array}$ & $\begin{array}{l}\text { Intervention } \\
\text { Group } \\
(n=467)\end{array}$ & $\mathbf{P}$ & $\begin{array}{l}\text { Control } \\
\text { Group } \\
(n=325)\end{array}$ & $\begin{array}{l}\text { Intervention } \\
\text { Group } \\
(n=325)\end{array}$ & $\mathbf{P}$ \\
\hline \multirow[t]{2}{*}{ Gender, n, \% } & Male & $333(80.4)$ & $36 \mid(77.3)$ & 0.256 & $259(79.7)$ & $250(76.9)$ & \multirow[t]{2}{*}{0.446} \\
\hline & Female & $81(19.6)$ & $106(22.7)$ & & $66(20.3)$ & $75(23.1)$ & \\
\hline \multirow[t]{2}{*}{ Age, n, \% } & $\leqq 60$ year & $242(58.5)$ & $293(62.7)$ & 0.193 & $195(60.0)$ & $186(57.2)$ & \multirow[t]{2}{*}{0.524} \\
\hline & $>60$ year & $172(4 \mid .5)$ & $174(37.3)$ & & I $30(40.0)$ & $139(42.8)$ & \\
\hline \multirow[t]{3}{*}{ BMI, n, kg/m } & $<18.5$ & $38(9.2)$ & $4 \mathrm{I}(8.8)$ & 0.732 & $33(10.2)$ & $30(9.2)$ & \multirow[t]{3}{*}{0.794} \\
\hline & $18.5-25$ & $279(67.4)$ & $326(69.8)$ & & $218(67.1)$ & $226(69.5)$ & \\
\hline & $\geqq 25$ & $97(23.4)$ & $100(21.4)$ & & $74(22.8)$ & $69(21.2)$ & \\
\hline \multirow[t]{2}{*}{ Smoking, n, \% } & Yes & $109(26.3)$ & 147 (3I.5) & 0.093 & $93(28.6)$ & $97(29.8)$ & \multirow[t]{2}{*}{0.796} \\
\hline & No & $305(73.7)$ & $320(68.5)$ & & $232(71.4)$ & $228(70.2)$ & \\
\hline \multirow[t]{2}{*}{ Hypertension, n, \% } & Yes & 47 ( 11.4$)$ & $68(14.6)$ & 0.158 & $39(12.0)$ & $40(12.3)$ & \multirow[t]{2}{*}{1.000} \\
\hline & No & $367(88.6)$ & $399(85.4)$ & & $286(88.0)$ & $285(87.7)$ & \\
\hline \multirow[t]{2}{*}{ Diabetes mellitus, $n, \%$} & Yes & $20(4.8)$ & $27(5.8)$ & 0.531 & $19(5.8)$ & $17(5.2)$ & \multirow[t]{2}{*}{0.864} \\
\hline & No & $394(95.2)$ & $440(94.2)$ & & $306(94.2)$ & $308(94.8)$ & \\
\hline \multirow[t]{2}{*}{ Coronary heart disease, n, \% } & Yes & $15(3.6)$ & $18(3.9)$ & 0.857 & $12(3.7)$ & $12(3.7)$ & \multirow[t]{2}{*}{1.000} \\
\hline & No & $399(96.4)$ & $449(96.1)$ & & $313(96.3)$ & $313(96.3)$ & \\
\hline \multirow[t]{4}{*}{ ASA classification, $n, \%$} & Class I & $9(2.2)$ & $43(9.2)$ & $<0.001$ & $9(2.8)$ & $17(5.2)$ & \multirow[t]{4}{*}{0.228} \\
\hline & Class 2 & $301(72.7)$ & $336(71.9)$ & & $252(77.5)$ & $232(7 \mid .4)$ & \\
\hline & Class 3 & $103(24.9)$ & $87(18.6)$ & & $63(19.4)$ & $75(23.1)$ & \\
\hline & Class 4 & $\mathrm{I}(0.2)$ & $\mathrm{I}(0.2)$ & & $\mathrm{I}(0.3)$ & $\mathrm{I}(0.3)$ & \\
\hline \multirow[t]{2}{*}{ Operative approach, n, \% } & Open surgery & $315(76.1)$ & $283(60.6)$ & $<0.001$ & $229(70.5)$ & $228(70.2)$ & \multirow[t]{2}{*}{1.000} \\
\hline & $\begin{array}{l}\text { Laparoscopic } \\
\text { surgery }\end{array}$ & 99 (23.9) & $184(39.4)$ & & $96(29.5)$ & $97(29.8)$ & \\
\hline \multirow[t]{3}{*}{ Type of gastrectomy, n, \% } & Proximal & $85(20.5)$ & 71 (15.2) & 0.010 & $56(17.2)$ & $56(17.2)$ & \multirow[t]{3}{*}{0.967} \\
\hline & Distal & $180(43.5)$ & $184(39.4)$ & & |36 (4I.8) & $133(40.9)$ & \\
\hline & Total & $149(36.0)$ & $212(45.4)$ & & $133(40.9)$ & $136(4 \mid .8)$ & \\
\hline \multirow[t]{4}{*}{ Tumor location, n, \% } & Upper third & $|3|(3 \mid .6)$ & $129(27.6)$ & 0.512 & $102(31.4)$ & $89(27.4)$ & \multirow[t]{4}{*}{0.603} \\
\hline & Middle third & $100(24.2)$ & 127 (27.2) & & $79(24.3)$ & $90(27.7)$ & \\
\hline & Lower third & $140(33.8)$ & $166(35.5)$ & & III (34.2) & $116(35.7)$ & \\
\hline & Entire & $43(10.4)$ & $45(9.6)$ & & $33(10.2)$ & $90(9.2)$ & \\
\hline \multirow[t]{2}{*}{ Tumor size(cm), n, \% } & $\leqq 5 \mathrm{~cm}$ & $289(69.8)$ & 331 (70.9) & 0.728 & $229(70.5)$ & $234(72)$ & \multirow[t]{2}{*}{0.729} \\
\hline & $>5 \mathrm{~cm}$ & $125(30.2)$ & $136(29.1)$ & & $96(29.5)$ & $91(28)$ & \\
\hline
\end{tabular}

(Continued) 
Table I (Continued).

\begin{tabular}{|c|c|c|c|c|c|c|c|}
\hline & & \multicolumn{3}{|c|}{ Before Matching $(n=88 I)$} & \multicolumn{3}{|c|}{ After Matching $(n=650)$} \\
\hline & & $\begin{array}{l}\text { Control } \\
\text { Group } \\
(n=4 \mid 4)\end{array}$ & $\begin{array}{l}\text { Intervention } \\
\text { Group } \\
(n=467)\end{array}$ & $\mathbf{P}$ & $\begin{array}{l}\text { Control } \\
\text { Group } \\
(n=325)\end{array}$ & $\begin{array}{l}\text { Intervention } \\
\text { Group } \\
(n=325)\end{array}$ & $\mathbf{P}$ \\
\hline \multirow[t]{2}{*}{ Differentiated grade, $n, \%$} & Well\&moderately & $189(45.7)$ & 177 (37.9) & 0.020 & $133(40.9)$ & I $36(4 \mid .8)$ & \multirow[t]{2}{*}{0.874} \\
\hline & Poor & $225(54.3)$ & $290(62.1)$ & & $192(59.1)$ & $189(58.2)$ & \\
\hline \multirow[t]{5}{*}{ Tumor depth, n, \% } & TI & $74(17.9)$ & $70(15.0)$ & 0.016 & $49(15.1)$ & $57(17.5)$ & \multirow[t]{5}{*}{0.688} \\
\hline & $\mathrm{T} 2$ & $99(23.9)$ & $82(17.6)$ & & 71 (2I.8) & $61(18.8)$ & \\
\hline & T3 & $232(56.0)$ & 291 (62.3) & & $196(60.3)$ & $194(59.7)$ & \\
\hline & T4a & $7(1.7)$ & $20(4.3)$ & & $7(2.2)$ & II (3.4) & \\
\hline & $\mathrm{T} 4 \mathrm{~b}$ & $2(0.5)$ & $4(0.9)$ & & $2(0.6)$ & $2(0.6)$ & \\
\hline \multirow[t]{5}{*}{ LNM, n, \% } & No & $168(40.6)$ & $159(34.0)$ & 0.040 & $117(36.0)$ & $12 \mid(37.2)$ & \multirow[t]{5}{*}{0.738} \\
\hline & NI & $105(25.4)$ & $110(23.6)$ & & $85(26.2)$ & $80(24.6)$ & \\
\hline & N2 & $64(15.5)$ & $76(16.3)$ & & $53(16.3)$ & $49(15.1)$ & \\
\hline & N3a & $59(14.3)$ & $83(17.8)$ & & $53(16.3)$ & $50(15.4)$ & \\
\hline & N3b & $18(4.3)$ & $39(8.4)$ & & $17(5.2)$ & $25(7.7)$ & \\
\hline \multirow[t]{6}{*}{ Tumor stage, n, \% } & la & $62(15.0)$ & $56(12.0)$ & 0.025 & $42(12.9)$ & $47(14.5)$ & \multirow[t]{6}{*}{0.713} \\
\hline & $\mathrm{lb}$ & $2(0.5)$ & I $(0.2)$ & & I (0.3) & I $(0.3)$ & \\
\hline & Ila & $138(33.3)$ & I34 (28.7) & & $97(29.8)$ & $99(30.5)$ & \\
\hline & Ilb & $89(21.5)$ & $93(19.9)$ & & $74(22.8)$ & $63(19.4)$ & \\
\hline & IIlb & $69(16.7)$ & 107 (22.9) & & $62(19.1)$ & $69(21.2)$ & \\
\hline & IIllc & $3(0.7)$ & $15(3.2)$ & & $3(0.9)$ & $7(2.2)$ & \\
\hline
\end{tabular}

within 30 days, including pneumonia, ileus, anastomosis leakage, abdominal bleeding, postoperative abdominal infection, wound complication, unplanned reoperation, and mortality.

The detailed mechanism of action of SSCs on postoperative complications remains unclear. It may be caused by the Hawthorne effect owing to the inability to conduct randomized and double-blind controlled trials in clinical studies on SSCs, i.e., patients and medical staff who implement SSC will have better job performance because of the effect of being observed..$^{5}$ Alternatively, it may be related to safety culture and the atmosphere among the surgical team (surgeons, anesthesiologists, nurses, etc.) after SSC implementation, ${ }^{17-19}$ as well as communication among team members. ${ }^{20}$ However, these reasons cannot logically explain how SSCs help improve the quality of

Table 2 Comparison Of Surgery-Related Parameters After Matching

\begin{tabular}{|l|l|l|l|}
\hline & Control Group $(\mathbf{n}=325)$ & Intervention Group $(\mathbf{n}=325)$ & P value \\
\hline Operation time, min, mean \pm SD & $224.70 \pm 106.58$ & $218.98 \pm 74.05$ & 0.427 \\
Blood loss, mL, mean \pm SD & $226.03 \pm 159.41$ & $248.58 \pm 201.55$ & 0.114 \\
Transfusion, yes, n, \% & $59(18.2)$ & $73(22.5)$ & 0.205 \\
Length of postoperative stay, day, mean \pm SD & $11.02 \pm 3.30$ & $9.64 \pm 2.67$ & $<0.001$ \\
Hospital charges, IOK RMB, mean \pm SD & $3.74 \pm 0.98$ & $3.84 \pm 0.79$ & 0.153 \\
\hline
\end{tabular}


Table 3 Comparison Of Postoperative Complications After Matching

\begin{tabular}{|l|l|l|l|}
\hline & Control Group $(\mathbf{n}=\mathbf{3 2 5})$ & Intervention Group $(\mathbf{n}=\mathbf{3 2 5})$ & P value \\
\hline Postoperative complications, $\mathrm{n}, \%$ & $32(9.8)$ & $32(9.8)$ & 1.000 \\
Pneumonia, n, \% & $13(4.0)$ & $13(4.0)$ & 1.000 \\
lleus, $\mathrm{n}, \%$ & $3(0.9)$ & $6(1.8)$ & 0.502 \\
Anastomosis leakage, n, \% & $4(1.2)$ & $5(1.5)$ & 1.000 \\
Abdominal bleeding, n, \% & $8(2.5)$ & $6(1.8)$ & 0.787 \\
Postoperative abdominal infection, n,\% & $4(1.2)$ & $2(0.6)$ & 0.682 \\
Wound complication, n, \% & $7(2.2)$ & $6(1.8)$ & 1.000 \\
Unplanned reoperation, n, \% & $5(1.5)$ & $5(1.5)$ & 1.000 \\
Mortality, n, \% & $4(1.2)$ & $8(2.5)$ & 0.382 \\
\hline
\end{tabular}

Table 4 Univariate Analysis Of Risk Factors For Prognosis Of Gastric Cancer After Matching

\begin{tabular}{|c|c|c|c|}
\hline \multicolumn{2}{|l|}{ Prognostic Factors } & \multirow{2}{*}{$\begin{array}{l}\boldsymbol{\beta}, \mathbf{9 5} \% \mathrm{Cl} \text { (Low To High) } \\
\text { Reference }\end{array}$} & \multirow{3}{*}{$\begin{array}{l}\text { P value } \\
0.7780\end{array}$} \\
\hline Gender & Male & & \\
\hline & female & I.IO $(0.58,2.08)$ & \\
\hline \multirow[t]{2}{*}{ Age } & $\leqq 60$ year & Reference & \multirow[b]{2}{*}{0.0836} \\
\hline & $>60$ year & $0.63(0.38,1.06)$ & \\
\hline \multirow[t]{3}{*}{ BMI } & $<18.5$ & Reference & \\
\hline & $18.5-25$ & $0.90(0.34,2.36)$ & 0.8232 \\
\hline & $\geqq 25$ & $0.53(0.19,1.48)$ & 0.2266 \\
\hline Smoking & Yes & Reference & \multirow[b]{2}{*}{0.5075} \\
\hline & No & $1.21(0.69,2.09)$ & \\
\hline \multirow[t]{2}{*}{ Hypertension } & Yes & Reference & \multirow[b]{2}{*}{0.0386} \\
\hline & No & $2.01(1.04,3.89)$ & \\
\hline \multirow[t]{2}{*}{ Diabetes mellitus } & Yes & Reference & \multirow[b]{2}{*}{0.7934} \\
\hline & No & $1.15(0.39,3.37)$ & \\
\hline \multirow[t]{2}{*}{ Coronary heart disease, } & Yes & Reference & \multirow[b]{2}{*}{0.2606} \\
\hline & No & $1.89(0.62,5.70)$ & \\
\hline \multirow[t]{4}{*}{ ASA classification } & Class I & Reference & \\
\hline & Class 2 & $1.34(0.39,4.64)$ & 0.6464 \\
\hline & Class 3 & $0.87(0.24,3.19)$ & 0.8333 \\
\hline & Class 4 & $276284.34(0.00$, Inf $)$ & 0.9840 \\
\hline \multirow[t]{2}{*}{ Operative approach } & Open surgery & Reference & \multirow[b]{2}{*}{0.5642} \\
\hline & Laparoscopic surgery & $1.19(0.66,2.12)$ & \\
\hline \multirow[t]{3}{*}{ Type of gastrectomy } & Proximal & Reference & \\
\hline & Distal & $0.68(0.28,1.63)$ & 0.3873 \\
\hline & Total & $0.48(0.20,1.11)$ & 0.0867 \\
\hline Tumor location & Upper third & Reference & \\
\hline
\end{tabular}


Table 4 (Continued).

\begin{tabular}{|c|c|c|c|}
\hline \multicolumn{2}{|l|}{ Prognostic Factors } & \multirow{2}{*}{$\begin{array}{l}\boldsymbol{\beta}, \mathbf{9 5} \% \mathrm{Cl} \text { (Low To High) } \\
0.74(0.38,1.42)\end{array}$} & \multirow{2}{*}{$\frac{P \text { value }}{0.3614}$} \\
\hline & Middle third & & \\
\hline & Lower third & $1.46(0.73,2.92)$ & 0.2886 \\
\hline & Entire & $0.88(0.35,2.21)$ & 0.7919 \\
\hline \multirow[t]{2}{*}{ Tumor size } & $\leqq 5 \mathrm{~cm}$ & Reference & \multirow[b]{2}{*}{0.9046} \\
\hline & $>5 \mathrm{~cm}$ & $1.04(0.58,1.84)$ & \\
\hline \multirow[t]{2}{*}{ Differentiated grade } & Well \& moderately & Reference & \multirow[b]{2}{*}{0.5020} \\
\hline & Poor & I.19(0.7I, 2.0I) & \\
\hline \multirow[t]{5}{*}{ Tumor depth } & TI & Reference & \\
\hline & $\mathrm{T} 2$ & $\mathrm{I} .40(0.6 \mathrm{I}, 3.2 \mathrm{I})$ & 0.4290 \\
\hline & T3 & I.37(0.70, 2.70) & 0.3550 \\
\hline & $\mathrm{T} 4 \mathrm{a}$ & $0.70(0.18,2.75)$ & 0.6080 \\
\hline & $\mathrm{T} 4 \mathrm{~b}$ & $804855.56(0.00, \operatorname{lnf})$ & 0.9851 \\
\hline \multirow[t]{5}{*}{ LNM } & No & Reference & \\
\hline & $\mathrm{NI}$ & $1.74(0.90,3.36)$ & 0.1011 \\
\hline & N2 & $2.58(1.04,6.35)$ & 0.0400 \\
\hline & N3a & $1.68(0.77,3.65)$ & 0.1897 \\
\hline & N3b & $3.22(0.74,13.96)$ & 0.1182 \\
\hline \multirow[t]{7}{*}{ Tumor stage } & la & Reference & \\
\hline & $\mathrm{lb}$ & $2677206.45(0.00$, Inf $)$ & 0.9930 \\
\hline & lla & $1.43(0.68,2.99)$ & 0.3493 \\
\hline & Ilb & $1.50(0.67,3.37)$ & 0.3227 \\
\hline & Illa & $3.46(1.08,11.09)$ & 0.0364 \\
\hline & Illb & $1.70(0.74,3.91)$ & 0.2152 \\
\hline & IIlc & $2677206.45(0.00, \mathrm{Inf})$ & 0.9844 \\
\hline \multirow[t]{2}{*}{ SSC } & Before & Reference & \multirow[b]{2}{*}{1.000} \\
\hline & After & $1.00(0.60,1.68)$ & \\
\hline
\end{tabular}

gastric cancer surgery. SSC implementation may help reduce human errors, such as patient errors, site errors, etc. However, the incidence of such errors in gastric cancer surgery is extremely low; there are no patient errors or surgical resection site errors during gastric cancer surgery in the history of our center. Given this perspective, a new checklist covering the entire perioperative period may be needed to reduce surgical complications for gastric cancer.

The negative findings of our study may be due to the compliance of implementation. In 2017, a prospective study by $\mathrm{Yu}$ et $\mathrm{al}^{21}$ showed a clear relationship between a reduction in postoperative complications and the extent to which SSC was followed after its implementation by the hospital, i.e., a higher compliance with the SSC leads to a more significant reduction in postoperative complications and vice versa. Considering that SSC implementation can be affected by many factors, the rate of implementation varies significantly. ${ }^{22}$ Thus, some researchers have suggested increasing the actual implementation rate by using an electronic $\mathrm{SSC}^{23}$ or other method. At the same time, it 
A.

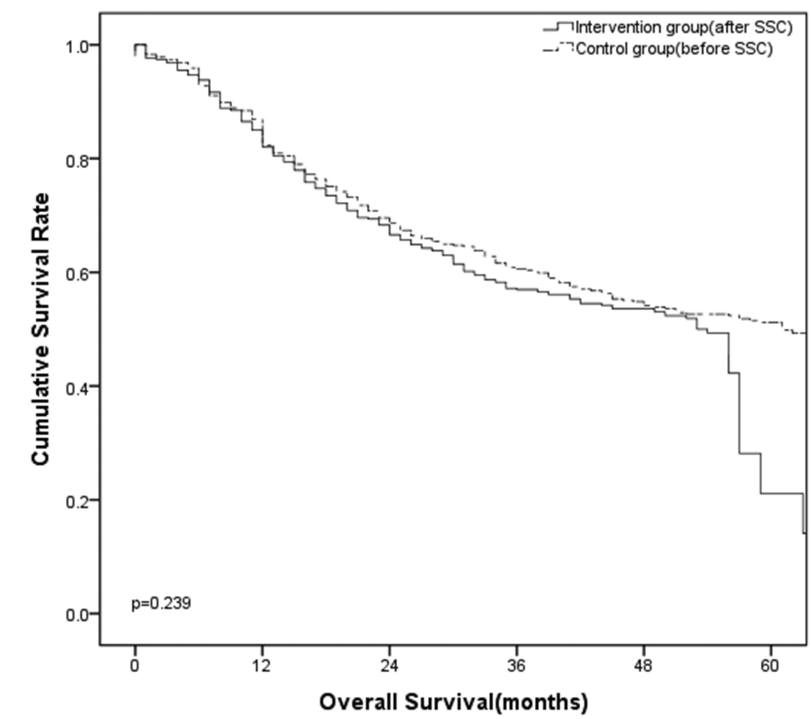

B.

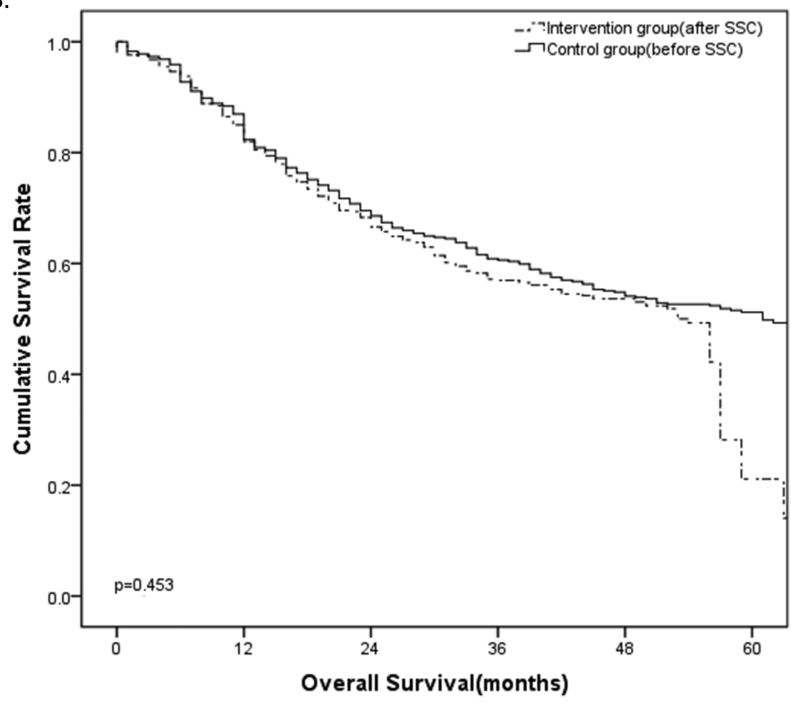

Figure 2 Overall survival of gastric cancer patients stratified by SSC. (A) Overall survival of gastric cancer patients before matching $(p=0.239)$. (B) Overall survival of gastric cancer patients after matching $(P=0.453)$.

is important to strengthen training ${ }^{24}$ and supervision ${ }^{25}$ for the use of SSCs.

Although previous research has shown that the implementation of SSCs may help reduce postoperative complications, there is no positive correlation between SSC and surgical prognosis. Similarly, no study has suggested that SSCs can improve the prognosis of gastric cancer surgery. The implementation of an SSC as a safety improvement measure is expected to reduce the complications of the operation. However, a high-quality operation does not necessarily mean lead to a good prognosis, which is more closely related to the patient's physical condition and staging. Our study showed that SSC had no effect on the prognosis of gastric cancer surgery. Nevertheless, SSCs have practical value.

This study is subject to several limitations. First, this study was a single-center study with a limited sample size, which may result in bias during analysis. A multicenter study with a larger sample size is needed to confirm our results. Second, our research design lacked data on SSC compliance. Although our center has very high SSC implementation rate, the effect of SSC compliance on the negative results of this study cannot be completely excluded. Third, as a retrospective analysis, our study lacked complete statistical data on all postoperative complications, including mild to moderate postoperative complications. More specific prospective studies are needed on the effect of SSC on varying degrees of complications, and more accurate subgroup analysis for postoperative complications should be performed on the basis of the Clavien-Dindo scoring system. Fourth, unfortunately the quality of postoperative follow-up is not good enough, that's a problem in the process of database construction in the early years. Some cases were lack of follow-up records and even lost follow-up. In the data collection process, the follow-up data was collected by electronic medical record system to minimize the data deviation as much as possible.

\section{Conclusion}

SSC implementation was associated with a decreased length of postoperative stay in gastric cancer patients but did not affect operation time, blood loss, blood transfusion, hospital charges, postoperative complications, or overall survival.

\section{Availability Of Data And Materials}

The datasets generated and/or analyzed during the current study are available from the corresponding author on reasonable request.

\section{Acknowledgments}

This study was supported in part by grants from the National Key Research and Development Program of China (NO. 2017YFC1311002, 2017YFC1311004) and the National Key Research and Development Program of Shann'xi Province, China (S2017-ZDYF-ZDXM-SF0181).

\section{Author Contributions}

All authors contributed to data analysis, drafting or revising the article, gave final approval of the version to be published, and agree to be accountable for all aspects of the work. 


\section{Disclosure}

The authors report no conflicts of interest in this work.

\section{References}

1. Bray F, Ferlay J, Soerjomataram I, Siegel RL, Torre LA, Jemal A Global cancer statistics 2018: GLOBOCAN estimates of incidence and mortality worldwide for 36 cancers in 185 countries. $C A$ Cancer J Clin. 2018;68:394-424. doi:10.3322/caac.21492

2. Chen W, Zheng R, Baade PD, et al. Cancer statistics in China 2015. CA Cancer J Clin. 2016;66:115-132. doi:10.3322/caac.21338

3. Statistical Information Center of the Ministry of Health. Statistics Yearbook of Chinese Health; 2013. [EB/OL], Available from: http:/ www.nhfpc.gov.cn/htmlfiles/zwgkzt/ptjnj/year2013/index2013.html. Accessed April 18, 2017.

4. Li Z, Liu Y, Bai B, Yu D, Lian B, Zhao Q. Surgical and long-term survival outcomes after laparoscopic and open total gastrectomy for locally advanced gastric cancer: a propensity score-matched analysis. World J Surg. 2019;43(2):594-603.

5. Haynes AB, Weiser TG, Berry WR, et al. A surgical safety checklist to reduce morbidity and mortality in a global population. $N$ Engl $J$ Med. 2009;360:491-499. doi:10.1056/NEJMsa0810119

6. O'Leary JD, Wijeysundera DN, Crawford MW. Effect of surgical safety checklists on pediatric surgical complications in Ontario. CMAJ. 2016;188:E191-E198. doi:10.1503/cmaj.151333

7. Urbach DR, Govindarajan A, Saskin R, Wilton AS, Baxter NN. Introduction of surgical safety checklists in Ontario, Canada. N Engl J Med. 2014;370:1029-1038. doi:10.1056/NEJMsa1308261

8. Morgan L, Hadi M, Pickering S, et al. The effect of teamwork training on team performance and clinical outcome in elective orthopaedic surgery: a controlled interrupted time series study. BMJ Open. 2015;5:e6216. doi:10.1136/bmjopen-2014-006216

9. de Jager E, McKenna C, Bartlett L, Gunnarsson R, Ho YH. Postoperative adverse events inconsistently improved by the World Health Organization Surgical Safety Checklist: a systematic literature review of 25 studies. World J Surg. 2016;40:1842-1858. doi:10.1007/ s00268-016-3519-9

10. Garcia-Paris J, Cohena-Jimenez M, Montano-Jimenez P, CórdobaFernández A. Implementation of the WHO "Safe Surgery Saves Lives" checklist in a podiatric surgery unit in Spain: a single-center retrospective observational study. Patient Saf Surg. 2015;9:29. doi:10.1186/s13037015-0068-3

11. Lepanluoma M, Rahi M, Takala R, Löyttyniemi E, Ikonen TS. Analysis of neurosurgical reoperations: use of a surgical checklist and reduction of infection-related and preventable complicationrelated reoperations. J Neurosurg. 2015;123:145-152. doi:10.3171/ 2014.12.JNS141077

12. Japanese Gastric Cancer Association. Japanese gastric cancer treatment guidelines 2010 (ver. 3). Gastric Cancer. 2011;14:113-123. doi:10.1007/s10120-011-0042-4
13. Gawande A, Thomas EJ, Zinner MJ, Brennan TA. The incidence and nature of surgical adverse events in Colorado and Utah in 1992. Surgery. 1999;126:66-75. doi:10.1067/msy.1999.98664

14. Kable AK, Gibberd RW, Spigelman AD. Adverse events in surgical patients in Australia. Int J Qual Health Care. 2002;14:269-276. doi:10.1093/intqhe/14.4.269

15. Papaconstantinou HT, Smythe WR, Reznik SI, Sibbitt S, WehbeJanek H. Surgical safety checklist and operating room efficiency: results from a large multispecialty tertiary care hospital. Am J Surg. 2013;206(853-859):859-860. doi:10.1016/j.amjsurg.2013.08.016

16. Lacassie HJ, Ferdinand C, Guzman S, Camus L, Echevarria GC. World Health Organization (WHO) surgical safety checklist implementation and its impact on perioperative morbidity and mortality in an academic medical center in Chile. Medicine (Baltimore). 2016;95 e3844. doi:10.1097/MD.0000000000004864

17. Zingiryan A, Paruch JL, Osler TM, Hyman NH. Implementation of the surgical safety checklist at a tertiary academic center: impact on safety culture and patient outcomes. Am J Surg. 2017;214:193-197. doi:10.1016/j.amjsurg.2016.10.027

18. Molina G, Jiang W, Edmondson L, et al. Implementation of the Surgical Safety Checklist in South Carolina Hospitals is associated with improvement in perceived perioperative safety. J Am Coll Surg. 2016;222:725-736. doi:10.1016/j.jamcollsurg.2015.12.052

19. Pires MP, Pedreira ML, Peterlini MA. Surgical safety in pediatrics: practical application of the pediatric surgical safety checklist. Rev Lat Am Enfermagem. 2015;23:1105-1112. doi:10.1590/0104-1169.0553. 2655

20. Cabral RA, Eggenberger T, Keller K, Gallison BS, Newman D. Use of a surgical safety checklist to improve team communication. Aorn J. 2016;104:206-216. doi:10.1016/j.aorn.2016.06.019

21. Yu X, Huang Y, Guo Q, Wang Y, Ma H, Zhao Y. Relaunch and Implementation of Operating Room Surgical Safety Checklist (RIORS) study group. Clinical motivation and the surgical safety checklist. Br J Surg. 2017;104:472-479. doi:10.1002/bjs. 10446

22. Sendlhofer G, Lumenta DB, Leitgeb $K$, et al. The gap between individual perception and compliance: a qualitative follow-up study of the surgical safety checklist application. PLoS One. 2016;11: e149212. doi:10.1371/journal.pone.0149212

23. Gitelis ME, Kaczynski A, Shear T, et al. Increasing compliance with the World Health Organization Surgical Safety Checklist-a regional health system's experience. Am J Surg. 2017;214:7-13. doi:10.1016/ j.amjsurg.2016.07.024

24. Melekie TB, Getahun GM. Compliance with surgical safety checklist completion in the operating room of University of Gondar Hospital, Northwest Ethiopia. BMC Res Notes. 2015;8:361. doi:10.1186/ s13104-015-1338-y

25. Sendlhofer G, Mosbacher N, Karina L, et al. Implementation of a surgical safety checklist: interventions to optimize the process and hints to increase compliance. PLoS One. 2015;10:e116926. doi:10. 1371/journal.pone.0116926

\section{Publish your work in this journal}

Cancer Management and Research is an international, peer-reviewed open access journal focusing on cancer research and the optimal use of preventative and integrated treatment interventions to achieve improved outcomes, enhanced survival and quality of life for the cancer patient.
The manuscript management system is completely online and includes a very quick and fair peer-review system, which is all easy to use. Visit http://www.dovepress.com/testimonials.php to read real quotes from published authors. 